\title{
1 Inducible Directed Evolution of Complex Phenotypes in
}

\section{Bacteria}

3 Ibrahim S. Al'Abri ${ }^{1}$, Daniel J. Haller ${ }^{1}$, Nathan Crook $^{1^{*}}$

4

$5{ }^{1}$ Department of Chemical and Biomolecular Engineering, North Carolina State University, Raleigh,

$6 \quad$ North Carolina, USA.

$7 \quad$ *To whom correspondence should be addressed: nccrook@ncsu.edu

\section{Abstract:}

9 Directed evolution is a powerful method for engineering biology in the absence of detailed 10 sequence-function relationships. To enable directed evolution of complex phenotypes encoded 11 by multigene pathways, we require large library sizes for DNA sequences $>5-10 \mathrm{~kb}$ in length, 12 elimination of genomic hitchhiker mutations, and decoupling of diversification and screening steps.

13 To meet these challenges, we developed Inducible Directed Evolution (IDE), which uses a 14 temperate bacteriophage to package large plasmids and transfer them to naive cells after 15 intracellular mutagenesis. To demonstrate IDE, we evolved a 5-gene pathway from Bacillus licheniformis that accelerates tagatose catabolism in Escherichia coli, resulting in clones with 65\%

17 shorter lag times during growth on tagatose after only two rounds of evolution.

18 Main:

19 Many important phenotypes emerge from the interactions between multiple genes ${ }^{1,2}$. These

20 "complex" phenotypes have traditionally encompassed small molecule biosynthesis ${ }^{3}$, tolerance to 21 inhibitors ${ }^{4}$, and growth in new habitats ${ }^{5}$. However, advances in synthetic biology and metabolic 
22

23

24

25

engineering have revealed that even supposedly "simple" phenotypes, such as production of a recombinant protein, become complex as higher performance is desired. This is because auxiliary cellular functions, such as chaperone proteins, cell wall synthesis, and secretion machinery can become limiting in these contexts ${ }^{6-8}$. Clearly, engineering these "systems-level" phenotypes requires systems-level techniques.

In bacteria, complex phenotypes can be accessed via adaptive evolution ${ }^{5,9,10}$ or iterative genomewide expression perturbation screens (e.g. asRNA ${ }^{11,12}$, and CRISPRi/a ${ }^{13-15}$ ). One downside of adaptive evolution is the accumulation of genomic hitchhiker mutations, a feature that is particularly troublesome for biosensor-coupled screens and that makes learning from these experiments very time-consuming. For asRNA and CRISPRi/a, the researcher is limited to sampling changes to expression space, rather than the much larger space of protein bioactivity.

For these reasons, directed evolution is useful because it directs mutations to defined DNA sequences and samples a much wider sequence space. However, due to the limited length of DNA that can be evolved using most methods, it has been difficult to apply directed evolution to complex phenotypes. For example, traditional error-prone PCR-based libraries are effectively limited to sequences $<10 \mathrm{~kb}$ in length due to reductions in polymerase processivity, cloning efficiency, and transformation rate above this size. Although recent methods for directed evolution in bacteria have eliminated many hands-on steps via the use of filamentous phage (e.g. phageassisted continuous and non-continuous evolution (PACE, PANCE) ${ }^{16-18}$, phagemid-assisted continuous evolution (PACEmid) ${ }^{19}$ and Phage-and-Robotics- Assisted Near-Continuous Evolution $\left.(\mathrm{PRANCE})^{20}\right)$, they are limited to small regions of DNA $(<5 \mathrm{~kb})$ and often couple the mutagenesis and screening steps. This is because the phage used in these techniques (M13) has a strict packaging limit $(5 \mathrm{~kb})$ and is engineered to replicate as soon as a certain threshold of 
47 biological activity has been reached $^{21}$. Indeed, recent implementations of PACE to evolve 48 multigene pathways $(7 \mathrm{~kb})^{22}$ have been limited to relatively small libraries $\left(\sim 10^{5}\right)$.

Here we add Inducible Directed Evolution, which overcomes these challenges, (IDE, Figure 1a)

51 to the directed evolution toolkit. IDE harnesses the large genomes of temperate phages (40-100

$52 \mathrm{~kb}$ ) to evolve large DNA segments ${ }^{23}$, avoids the accumulation of off-target genomic mutations,

53 and decouples mutagenesis and screening steps. The IDE workflow is both simple and flexible.

54 Pathways of interest are assembled in a phagemid and transformed to a bacterium containing a

55 helper phage. The master regulator for this phage is placed under inducible control. Next,

56 mutagenesis is induced to create random mutations. Then, the phage lytic cycle is induced to

57 initiate phagemid packaging and cell lysis. The resulting phage particles can then be applied to an unmutated strain to start a screening step or another mutagenesis step. We demonstrated IDE

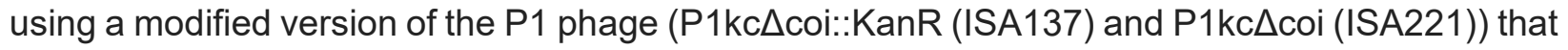
undergoes lysis in response to the addition of arabinose. Arabinose induces the expression of coi

61 to inhibit $c 1$, which is the master repressor of lysis ${ }^{24}$. This was achieved by knocking out coi, which

62 encodes a repressor of $c 1$, and placing it under the control of an arabinose-inducible promoter on

63 a P1 phagemid (PM) ${ }^{24}$. In the uninduced state, $c 1$ is expressed and maintains P1 lysogeny. After 64 coi induction, c1 can no longer maintain lysogeny, resulting in P1 particle production and lysis. 65 Inducible mutagenesis was achieved using a previously-described plasmid (MP6) ${ }^{25}$. 
a

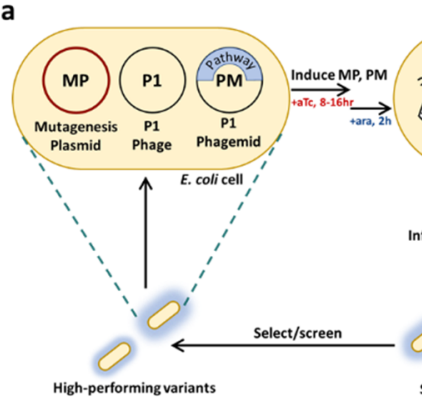

d

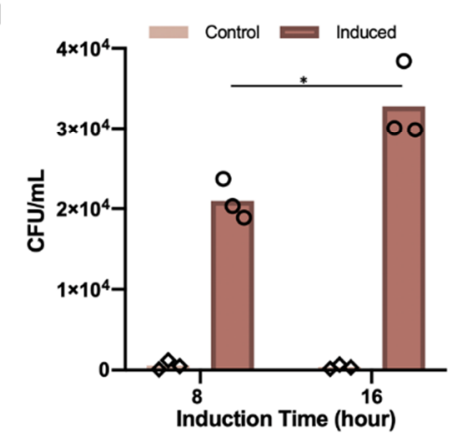

b

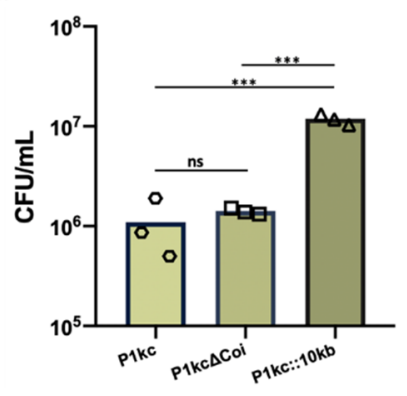

c

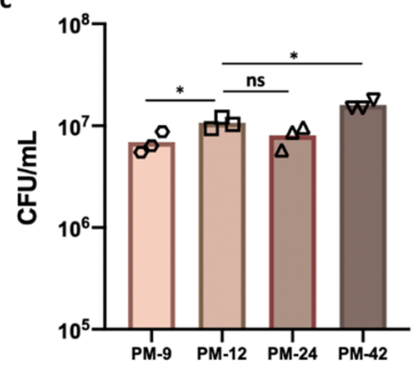

e

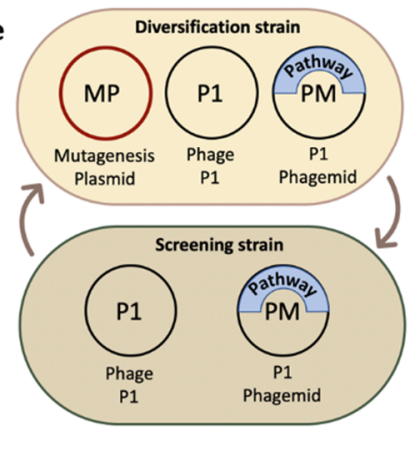

f

\begin{tabular}{|c|c|c|c|}
\cline { 2 - 4 } \multicolumn{1}{c|}{ Lysate } & C600 & MG1655 & Nissle \\
\hline C600 & $1.16 \mathrm{E}+07$ & $1.73 \mathrm{E}+07$ & $3.00 \mathrm{E}+07$ \\
\hline MG1655 & $3.07 \mathrm{E}+06$ & $4.20 \mathrm{E}+06$ & $3.73 \mathrm{E}+06$ \\
\hline Nissle & $7.60 \mathrm{E}+04$ & 0 & $1.60 \mathrm{E}+06$ \\
\hline
\end{tabular}

Figure 1. IDE overview and Optimization. a. IDE overview. b. Engineered P1kc (P1kc::10kb and $\mathrm{P} 1 \mathrm{kc} \Delta \mathrm{Coi}$ ) increases packaging/infection rates of phagemid compared to wild-type P1kc. c. The effect of insert size on phagemid transfer is negligible. PM-9 (9.7 kbp), PM-12 (12.8 kbp), PM-24 (24.0 kbp), and PM-42.0 (42.0 kbp) phagemid were packaged from the same strain (E. coli C600) containing $\mathrm{P} 1 \mathrm{kc}: 10 \mathrm{~kb}$ and same amount of phage lysate is used to infect wild-type $E$. coli $C 600$. d. Single stop codon reversion in $C m R$. A single stop codon was introduced to $C m R$ and reverted via AIDE, 3 replicate cultures were evolved to gain $C m R$ function e. Overview of using different $E$. coli strains in an IDE cycle for diversification and screening steps. f. Heat map summarizes infection/packaging rates $(\mathrm{CFU} / \mathrm{mL})$ of phage lysate produced from different $E$.coli strains (C600, MG1655 or Nissle) and used to infect the same 3 strains.

We first focused on P1 phagemid packaging and infection levels, as these metrics define the number of library members that can be passaged between evolutionary rounds and affect the explorable sequence space. Prior reports of lysate production using P1 were performed by diluting stationary, P1-containing cultures 100-fold into phage lysate medium (PLM) for $1 \mathrm{~h}$ at $37{ }^{\circ} \mathrm{C}$ followed by induction of lysis via addition of $13 \mathrm{mM}$ arabinose for $1-4$ hours at $37^{\circ} \mathrm{C}^{24,26}$. The 
77 We began by inducing phage production in a much larger E. coli C600 culture (OD 1, $\left.785^{\star} 10^{8} \mathrm{cells} / \mathrm{mL}\right)$ containing P1kc $\Delta$ coi (ISA221) and PM-12 (ISA012). The resulting lysate $(1 \mathrm{~mL})$

79 was applied to $3^{*} 10^{8}$ E. coli $\mathrm{C} 600$ cells containing $\mathrm{P} 1 \mathrm{kc} \Delta$ coi and plated on media selective for PM.

$806.1 \times 10^{5} \mathrm{PM}-$ containing cells were obtained. To increase this value, we first investigated the

81 composition of the media in which cell growth and infection was performed. $\mathrm{Ca}^{2+}\left(\mathrm{CaCl}_{2}\right)$ is

82 required for $\mathrm{P} 1$ adsorption to lipopolysaccharide (LPS), and adding $\mathrm{Mg}^{2+}\left(\mathrm{MgCl}_{2}\right)$ helps gram-

83 negative bacteria stabilize negatively charged lipopolysaccharides on the membrane ${ }^{27-29}$.

84 Therefore, $100 \mathrm{mM} \mathrm{MgCl}_{2}$ and $5 \mathrm{mM} \mathrm{CaCl}_{2}$ are commonly added to LB media (forming phage

85 lysate medium $(P L M))$ in studies of $P 1$ phage ${ }^{24,26}$. We hypothesized that increasing the concentration of $\mathrm{MgCl}_{2}$ and $\mathrm{CaCl}_{2}$ would enhance $\mathrm{P} 1$ infection rates. We found that when the

87 concentration of both salts in PLM is increased by $40 \%$, the number of PM-containing cells increased 2.2-fold to $1.3^{*} 10^{6}$. We called the new medium ePLM (Figure S1).

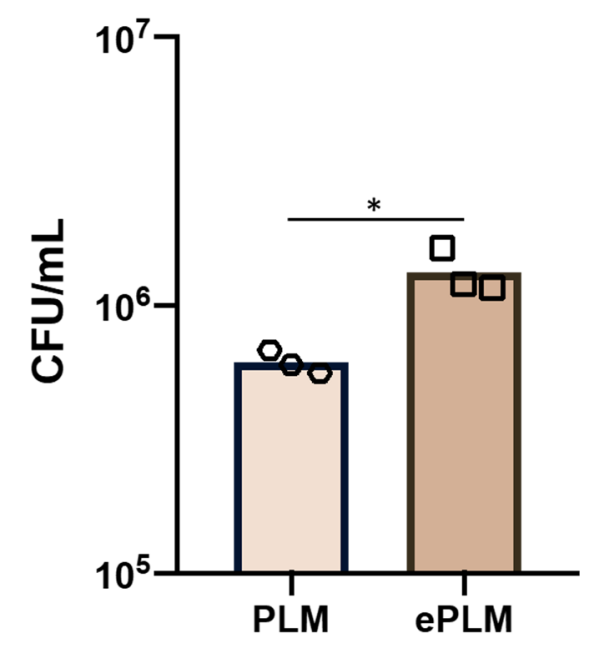

Figure S1. Increasing salt composition $\left(\mathrm{MgCl}_{2}\right.$ and $\left.\mathrm{CaCl}_{2}\right)$ in the growth medium influences phage infection rate. Each dot represents one biological replicate. 
We next varied the optical density to which the recipient cells were grown, while keeping the total

91 number of recipient cells constant. We found that our initial strategy of growing cells to an optical

92 density of 1.0 yielded the highest infection rates (Figure S2).

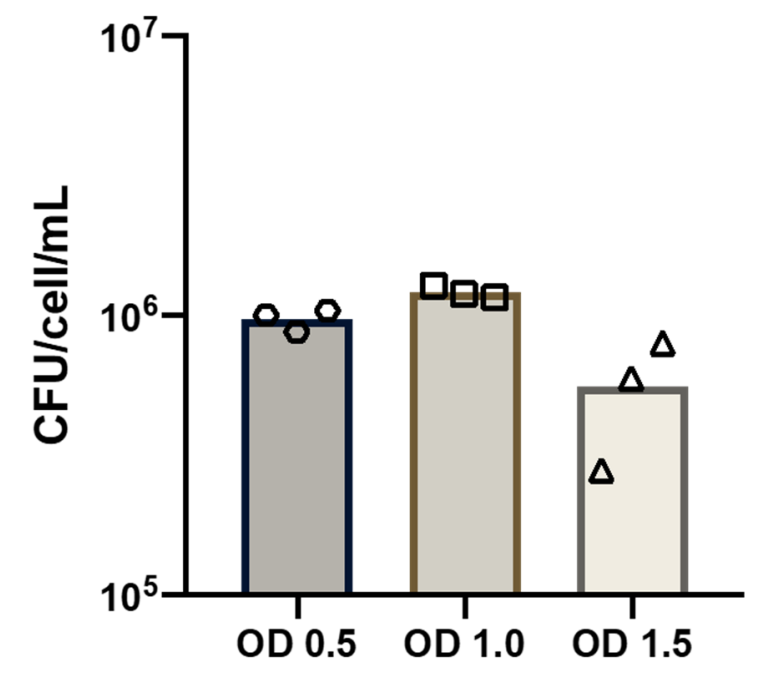

Figure S2. The physiological state of cells (E. coli C600) affects phagemid infection rate. Each dot represents one biological replicate.

Next, we hypothesized that reducing the ability of $\mathrm{P} 1 \mathrm{kc} \Delta \mathrm{coi}$ to be packaged in phagemids would

95 increase the proportion of particles carrying PM. We found that inserting $10 \mathrm{~kb}$ of yeast DNA into

96 P1kc (generating P1kc:10kb::KanR (ISA138) and P1kc::10kb (ISA222)) increased transferable

97 library size by an additional 9.2 -fold, to $1.2^{*} 10^{7}$ (Figure $1 \mathbf{b}, \mathbf{S 3}$ ). Similarly, we found that PM packaging was copy-number dependent, with lower PM copy numbers resulting in a reduced number of transduced cells (Figure S4). Taken together, over the course of these optimization methods ${ }^{24,30}$. 


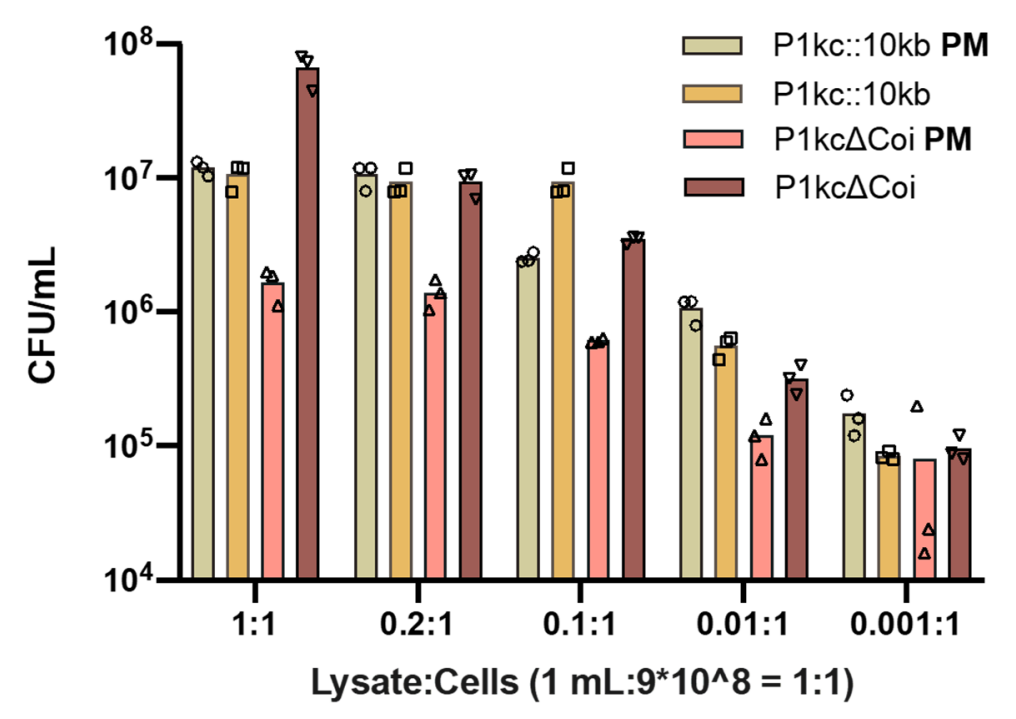

Figure S3. The ratio of lysate produced from strains containing phagemid and $\mathrm{P} 1:: 10 \mathrm{~kb}$ or $\mathrm{P} 1 \Delta \mathrm{Coi}$ affects the amount of the size of the library passaged in each cycle. Each dot represents one biological replicate.

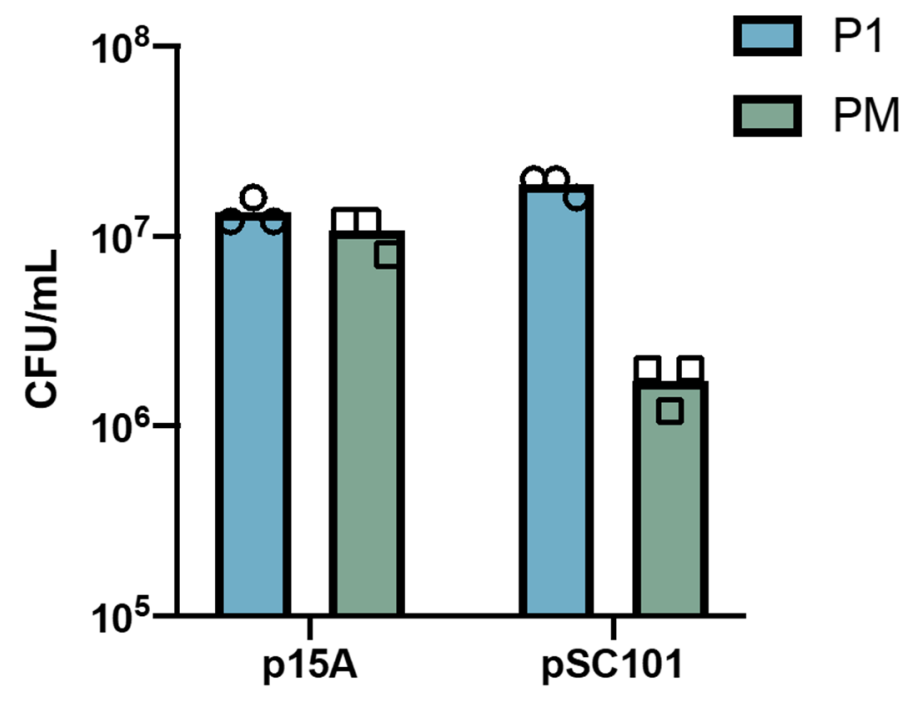

Figure S4. The copy number of phagemid impacts packaging and infection rates in the context of P1kc::10kb. Each dot represents one biological replicate.

104 Although we expected that increasing the amount of lysate applied to naive cells would increase

105 the number of transduced cells, we found that the ratio we had been using (lysate from $5^{*} 10^{8}$ cells applied to $3^{*} 10^{8}$ cells, defined as a ratio of $1: 1$ ) was past its saturation level, with reduced amounts of lysate providing similar values (Figure S3). Therefore, we varied the number of naive cells, 
holding the lysate volume constant. As expected, we observed a linear relationship between the number of naive cells and the number of infected cells (Figure S5), indicating that IDE library sizes can be easily increased by scaling up lysate and cell amounts.

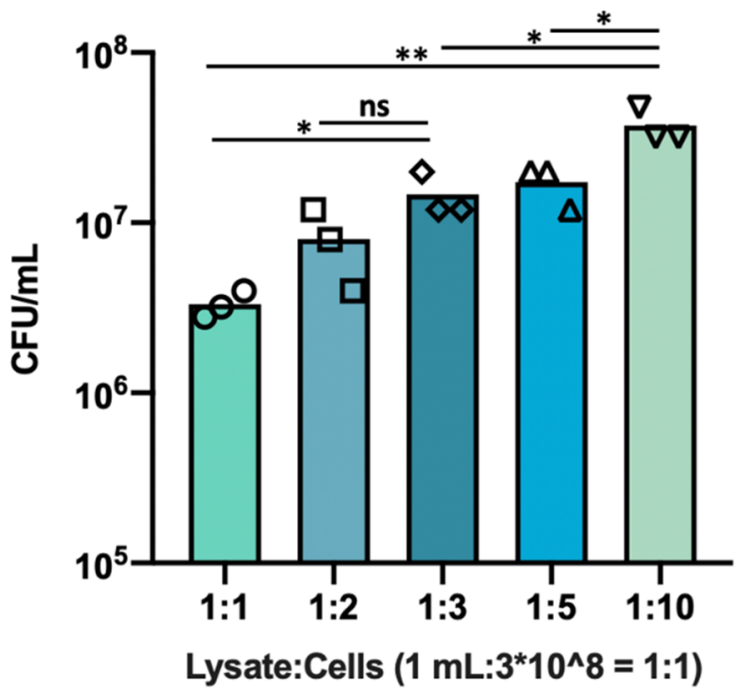

Figure S5. The amount of infected cells grown to OD 1 affect the size of the library passaged in each IDE cycle. 3 biological replicates of E. coli C600 cells were grown to OD 1 and concentrated to $1 \mathrm{x}, 2 \mathrm{x}$, $3 x, 5 x$ and $10 x$ and then infected with phage lvsate produced from E. coli $\mathrm{C} 600$.

Using these improved conditions, we investigated the effect of PM size on the number of transduced cells. We observed no reduction in library size with increasing cargo length, up to the largest phagemid we have tested (PM-42, $42.0 \mathrm{~kb}$ ) (Figure 1c). While we expect a substantial reduction of library size with phagemids larger than wild-type P1 (Figure 1b), this indicates that IDE is capable of efficiently evolving large multi-gene pathways. In comparison, when these phagemids were transformed via electroporation, the transformation efficiency decreased dramatically as the size of the phagemid increased, up to the size of the P1 genome (90kb) (Figure S6). In addition to the inefficiency of electroporating large plasmids, isolating and transforming large phagemids (e.g. via a kit) is costly and difficult to scale up compared to IDE (simple addition of inducer), making IDE a desirable approach for directed evolution of large phagemids. 


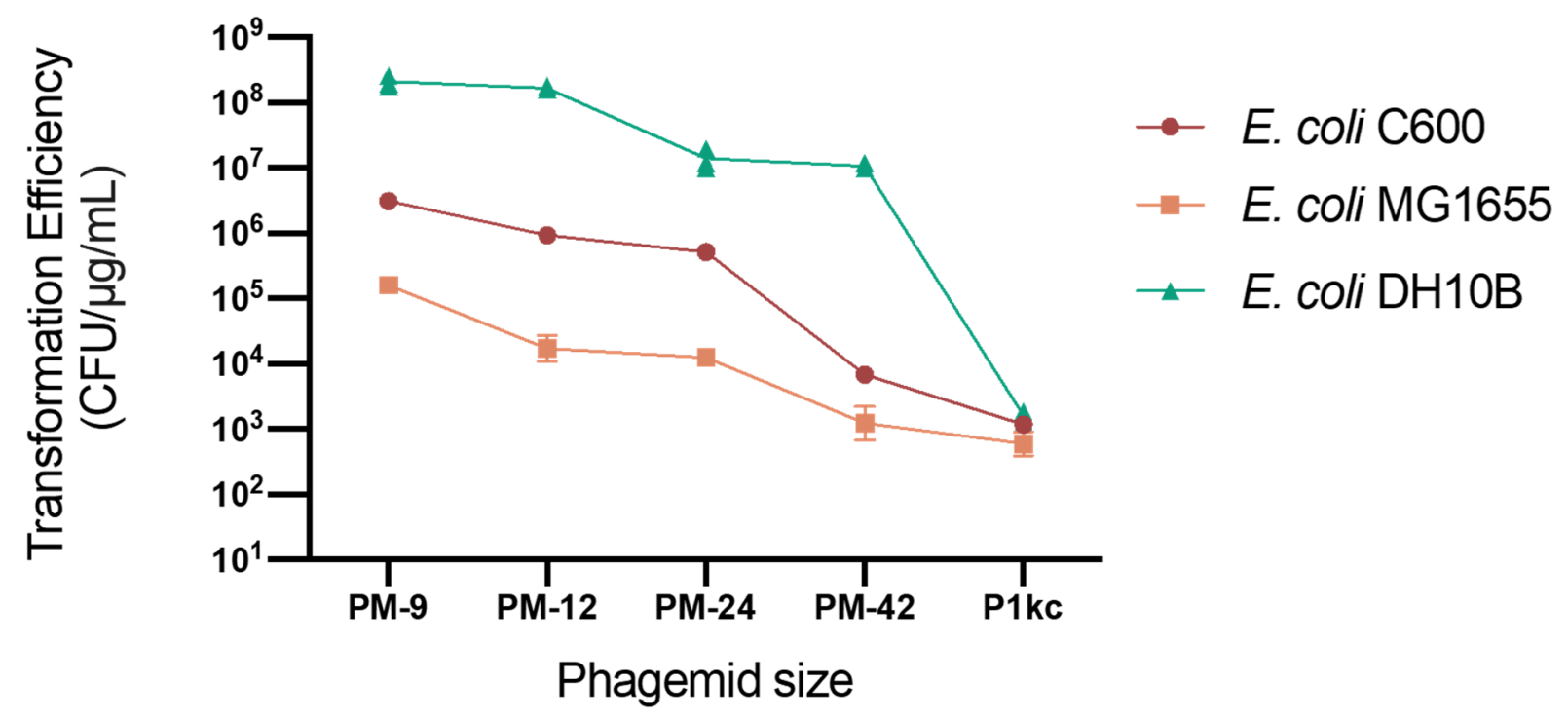

Figure S6. Transformation efficiency of E. coli $\mathrm{C} 600, \mathrm{MG} 1655$ and $\mathrm{DH} 10 \beta$ by different size phagemids. $1 \mu \mathrm{g}$ of each phagemid was transformed into freshly prepared electrocompetent cells.

124 Having established efficient transfer of phagemids between cell populations, we next asked 125 whether we could achieve mutations at rates sufficient for directed evolution. For this purpose, we modified a previously-reported plasmid enabling inducible mutagenesis (MP6) ${ }^{25}$. To ensure compatibility with PM, we switched this plasmid to an anhydrotetracycline (aTc)-inducible promoter and a kanamycin selection marker, forming aTc-MP. To test the mutation rate conferred by aTc-MP, we inserted a chloramphenicol resistance gene $(\mathrm{CmR})$ with one premature stop codon into PM (ISA308 and ISA311). We expected that inducing aTc-MP would randomly mutate

$131 \mathrm{CmR}$ and yield variants with the stop codon reverted to a functional codon. We observed time132 dependent increases in the number of $\mathrm{CmR}$-resistant cells after $16 \mathrm{~h}$ of induction (up to $5.4^{*} 10^{-5}$ 133 substitutions per base pair (bp), similar to the mutation rate of the original $\mathrm{MP}^{25}$ ), supporting the 134 notion that IDE enables tunable mutagenesis of defined DNA cargo (Figure 1d). Omitting the 135 inducer revealed that aTc-MP has a very tight off state, indicating that aTc-MP is suitable for 136 inclusion in cells during selection or screening steps. Additionally, we found that induction of aTc137 MP enabled the simultaneous reversion of two premature stop codons in $\mathrm{CmR}$ (ISA363) at a rate 

technique. The expected reversion mutations were confirmed via sequencing (Figure S7).
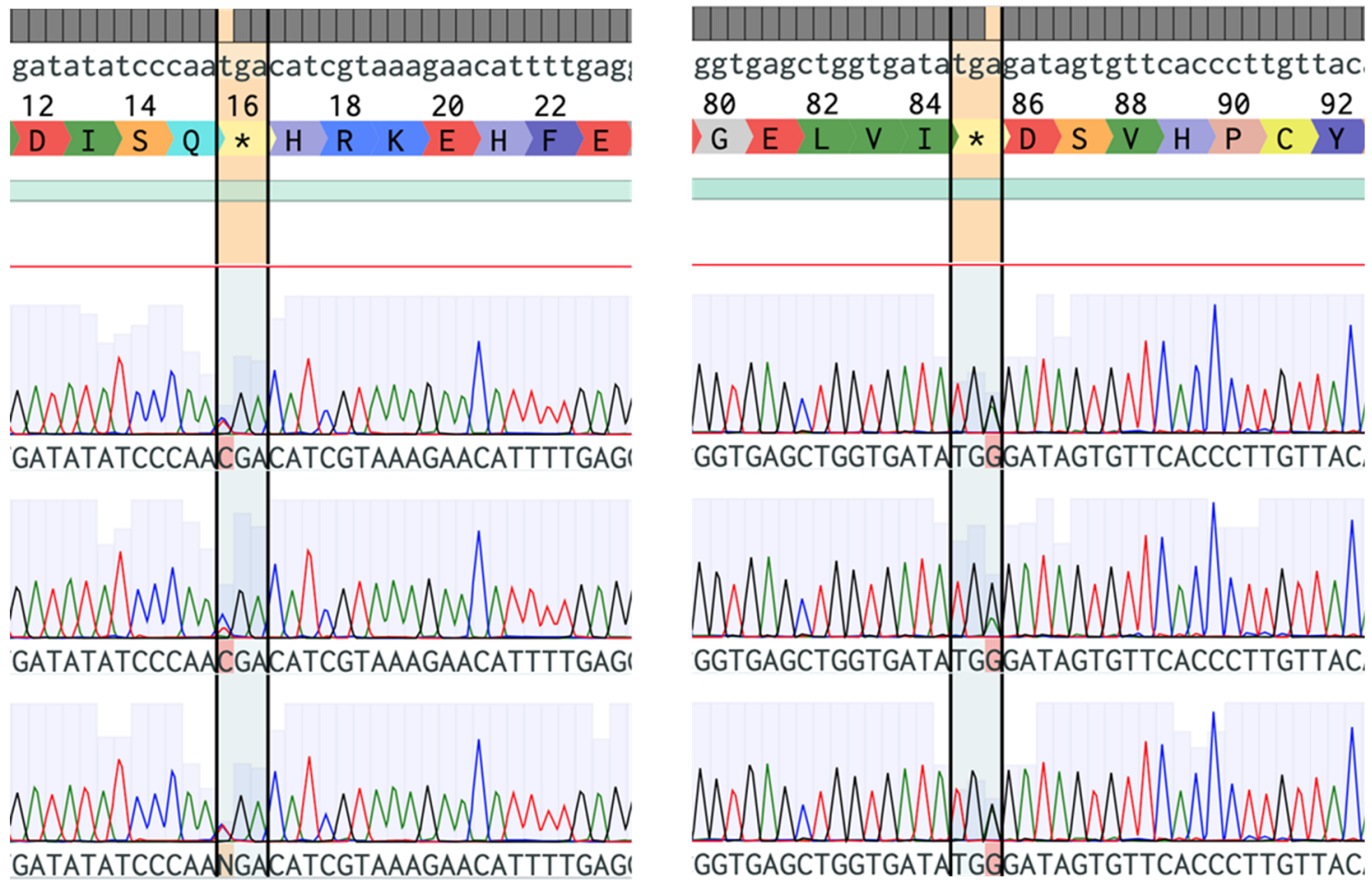

Figure S7. Sanger sequencing shows the reversion of two premature stop codons inserted in $\mathrm{CmR}$ at codons 16 and 85. Both codons were originally Trp (TGG). Double peaks indicate that the cells have two copies of the phagemids (one with stop codon and one with reverted stop codon).

Because mutagenesis and screening steps are decoupled in IDE, we hypothesized that the strain

142 that is used for screening does not have to be the same as the strain that is used for library

143 generation (Figure 1e). This would be beneficial if the ideal screening strain has a limited phage

144 production capacity. As examples, we found that phage lysate produced from $10^{8}$ E. coli C600

145 cells can passage $>10^{7}$ variants to E. coli MG1655 and E. coli Nissle 1917 (Figure 1f). On the

146 other hand, the same number of E. coli MG1655 and E. coli Nissle cells can only passage $4.9 \times 10^{6}$

147 and $6.9 \times 10^{4}$ variants back to $\mathrm{C600}$ (Figure 1g), respectively. These results indicate that $\mathrm{C} 600$ is

148 well-suited for production and packaging of large libraries, enabling these libraries to be screened

149 in a more appropriate strain, for example incorporating biosensors or production-coupled growth 150 circuits. 
152 Because phagemids are transferred to new cells after mutagenesis in IDE, we hypothesized that

153 recessive phenotypes would be easy to select for. Without passage to fresh cells, recessive

154 phenotypes would be difficult to observe due to the presence of multiple plasmid copies. We

155 therefore placed sfGFP on PM with medium copy number origin (p15A). Since production of

156 heterologous proteins incurs a fitness cost, cells containing inactivated sfGFP would outcompete

157 sfGFP-producing cells in a mixed culture ${ }^{32}$. Indeed, after 4 IDE cycles comprising mutagenesis, passage to fresh cells, and cell outgrowth, we found that almost all cells in the culture bore lossS8).

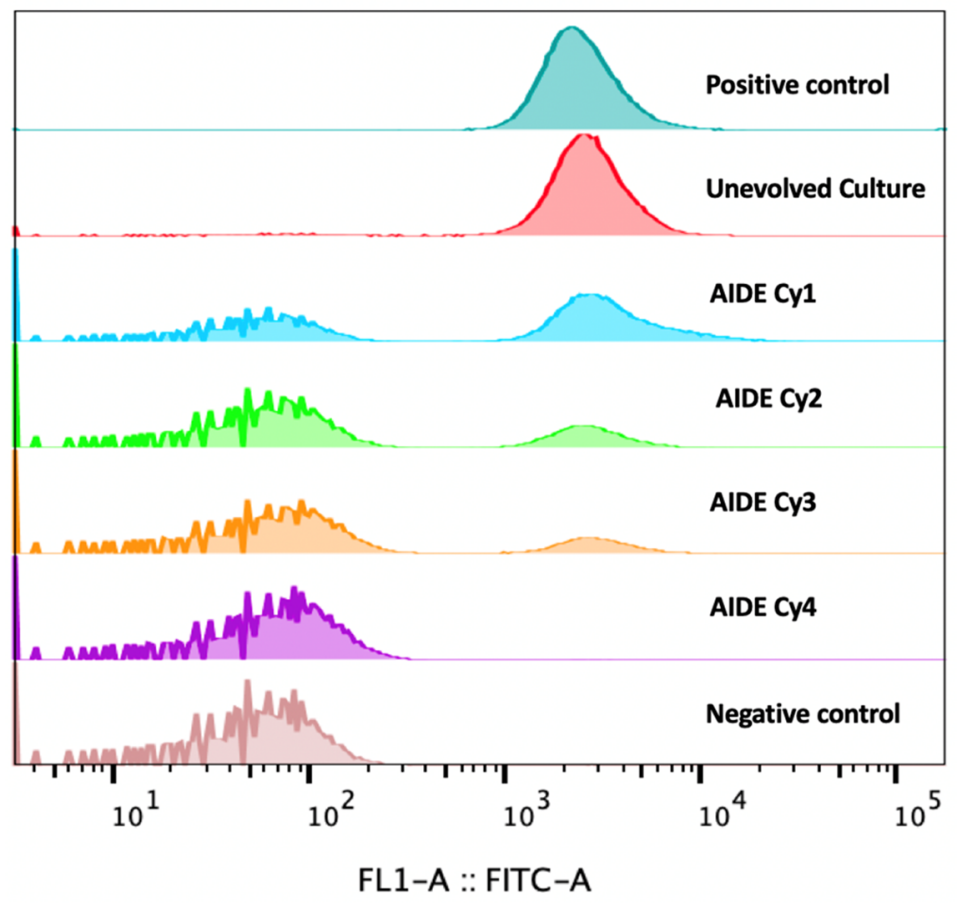

Figure S8. Directed Evolution of sfGFP using IDE. sfGFP phagemid was evolved to test the ability of IDE to passage recessive variants between cells across multiple rounds. The sfGFP phagemid went through 4 IDE cycles and was compared using flow cytometry to 1) a phagemid that was not mutagenized but that went through the rest of the IDE steps (unevolved culture), 2) negative controls (harboring an empty plasmid), and 3) positive controls (the starting plasmid). 


\begin{tabular}{|c|c|}
\hline Amino Acid Change & Sequence \\
\hline G33D & GGT $>$ GAC \\
\hline P56L & CCT -> CTC \\
\hline W57* & TGG -> TGA \\
\hline T62I & ACC -> ATC \\
\hline TY92* & TAT -> TAA \\
\hline A110V & GCG -> GTC \\
\hline G127D & GGC $>$ GAC \\
\hline Q157* & CAA $>$ TAA \\
\hline
\end{tabular}

Table S1. Detected mutations in the p15A-sfGFP phagemid after 4 IDE cycles. All mutations were detected in sfGFP. ${ }^{*}=$ stop codon.

165 To demonstrate IDE's capability to evolve a simplistic multi-gene phenotype, we assembled 166 sfGFP on a phagemid containing the pSC101 origin. We chose the pSC101 origin because of its 167 stringent Rep101-dependent replication mechanism and its low copy number ${ }^{34}$. In this setting, GFP fluorescence is controlled by at least 4 different genetic elements (the GFP coding sequence and its promoter, as well as Rep101 and its promoter). We wished to know which of these elements (or combination thereof) would lead to increased cellular fluorescence when mutated.

171 We found that after two sequential rounds of mutagenesis and passage to fresh cells, (Figure 172 2a), we were able to verify 7 highly fluorescent isolates out of 20 visually selected colonies. 173 Sequencing Rep101 and sfGFP in these 7 isolates yielded mutations exclusively in Rep101. To 174 separate these mutations from unknown mutations potentially present in other parts of PM, we 175 cloned these Rep101 variants into an unmutated PM-sfGFP vector and measured fluorescence 176 via flow cytometry. All variants yielded significantly higher fluorescence than wild-type (Figure 177 2b). Most of the Rep101 mutations present in these clones (R46W, M78I, E93G, E93K, K102E, 178 and $\mathrm{E} 115 \mathrm{~K})$ were previously found to increase the copy number of the $p S C 101$ origin $^{35}$, while one 179 highly beneficial variant (I94N) was novel. It is therefore likely that the increase in GFP production 180 in these isolates is due to an increased phagemid copy number. This result is reasonable because 

expression.
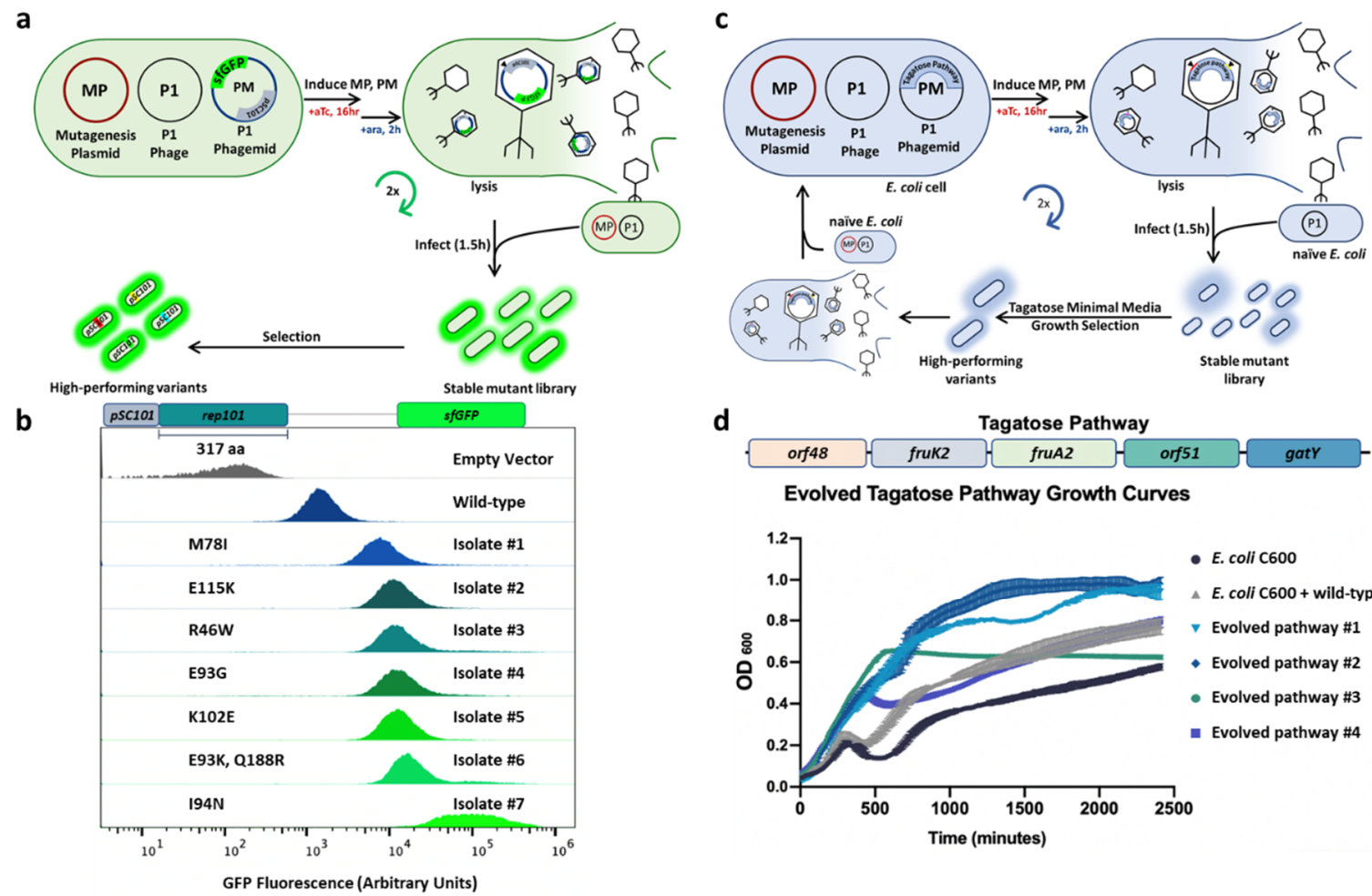

d
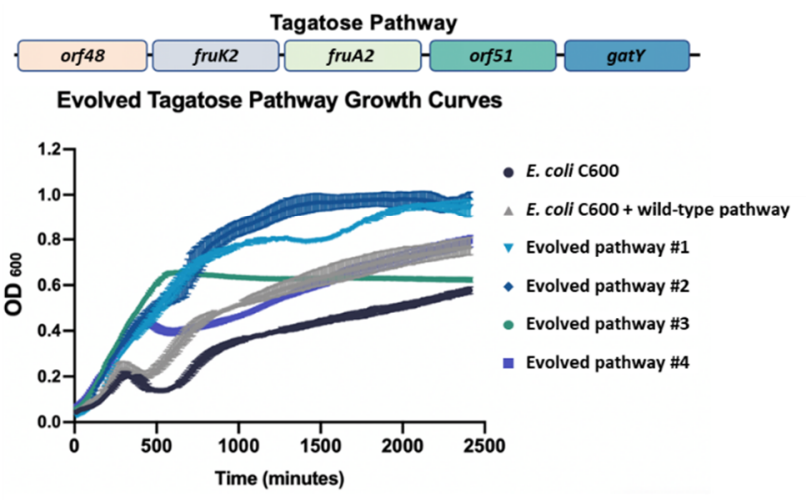

Figure 2. Directed evolution of complex phenotypes. a. Overview of evolving pSC101-sfGFP phagemid via IDE. b. Flow cytometry histograms of isolated and verified mutants compared to negative and positive controls. All mutations were detected in the pSC101 origin. c. Overview of evolving a tagatose pathway via IDE. d. Isolated tagatose pathway variants show improved growth on tagatose minimal media.

Finally, we applied IDE to improve a heterologous tagatose consumption pathway in $E$. coli

(Figure 2c). E. coli C600 natively consumes tagatose poorly, taking over 12.5 hours to reach an optical density (OD) of 0.25 in media containing tagatose as a sole carbon source from an initial OD of 0.05 . We chose a five-gene tagatose consumption pathway from Bacillus licheniformis for insertion in PM (Figure 2d) ${ }^{37}$. This pathway consists of orf48 (encoding a predicted transcriptional regulator in the murR/rpiR family), fruA2 and orf51 (encoding a predicted phosphotransferase 
192 (encoding a predicted kinase that converts tagatose 1-phosphate to tagatose 1,6-bisphosphate),

193 and gatY (encoding a predicted aldolase that converts tagatose 1,6-bisphosphate to

194 dihydroxyacetone phosphate and D-glyceraldehyde 3-phosphate). This pathway is therefore a

195 good test case for improving complex phenotypes with IDE, as it encodes different functions that

196 collectively elicit the phenotype of interest. Insertion of this pathway into PM yielded a C600 strain

197 with a $29 \%$ reduction in lag time, taking 8.8 hours to achieve an OD of 0.25 in tagatose media

198 (Figure 2d). We therefore expected that evolving this pathway would lead E. coli to consume

199 tagatose more efficiently. After 2 IDE cycles comprising mutagenesis, growth-based selection,

200 and transfer to fresh cells (Figure 2c), we selected for variants that grew faster in liquid tagatose

201 media than strains containing the unmutated pathway. We also performed a parallel selection

202 comprising the same steps, except that mutagenesis was not performed. Phagemids present in

203 cells surviving both selections were transferred to fresh E. coli C600, and the growth of strains

204 forming large colonies on tagatose minimal media agar plates was assayed in microtiter plates

205 (Figures S9 and S10). Strains from the mutagenic selection exhibited significantly higher growth

206 in tagatose minimal media than strains from the nonmutagenic selection $\left(p<10^{\wedge}-8\right.$, Student's T

207 test) (Figure S11). Eight strains from the mutagenic selection exhibiting the best combinations of

208 growth rate and final optical density were cloned into a wild type phagemid backbone (ISA012) to

209 verify increased growth (Figure S12a). Of these, four pathways conferred increased growth,

210 relative to the wild-type sequence (Figure 2c and $\mathbf{S 1 2 b ) . ~ A l l ~ f o u r ~ v a r i a n t s ~ e x h i b i t e d ~ s o m e ~}$

211 combination of higher optical density (strain E3 exhibited a 2.6-fold higher cell density at 500

212 minutes than a strain containing the unmutated pathway) and reduced lag time (strain E3

213 exhibited a $64 \%$ reduction in time to reach an optical density of 0.25 than a strain containing the

214 unmutated pathway). Mutations were identified in different genes, as shown in table S2. Isolates

$215 \mathrm{E} 1$ and E2 have mutations across two sets of 3 different genes (orf48, fruA2, and gatY for E1,

216 fruK2, fruA2, and gatY for E2), while isolates E3 and E4 share one mutation in the ribosome

217 binding site (RBS) of fruK2. This is the only mutation present in E4, while E3 contains two others 
218 (a silent mutation in fruK2 and a coding mutation in orf48) that together further increase growth.

219 The accumulation of fitness-enhancing mutations across multiple genes agrees with prior studies

220 pointing to the utility of a pathway-wide approach to directed evolution ${ }^{22,38}$.

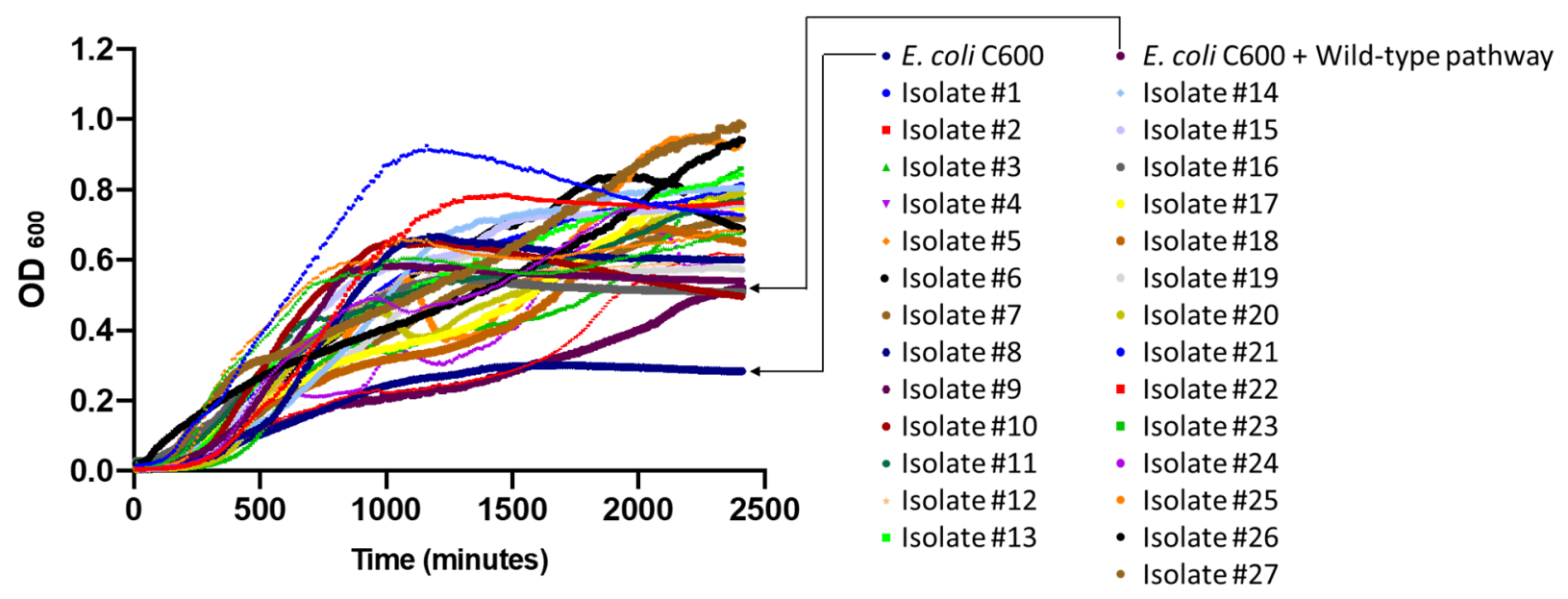

Figure S9. Growth curves of $\mathbf{2 7}$ tagatose variants. 27 colonies were picked from tagatose agar plates after 2 rounds of mutagenesis and selection. These colonies were grown in liquid tagatose media in a plate reader with wild-type E. coli $\mathrm{C} 600$ and E. coli $\mathrm{C} 600$ containing the wild-type pathway.

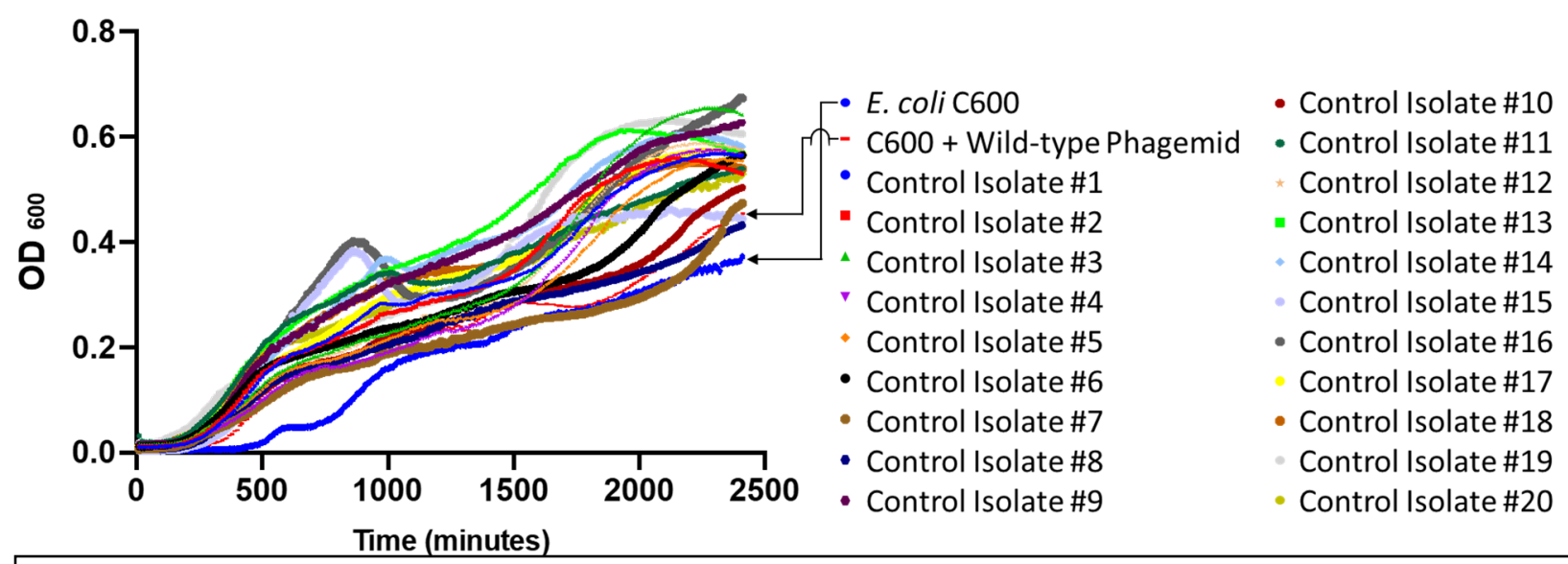

Figure S10. Growth curves of control colonies. 20 colonies were picked after 2 rounds of selection without mutagenesis. Colonies were picked from tagatose agar plates and grown in a plate reader. 


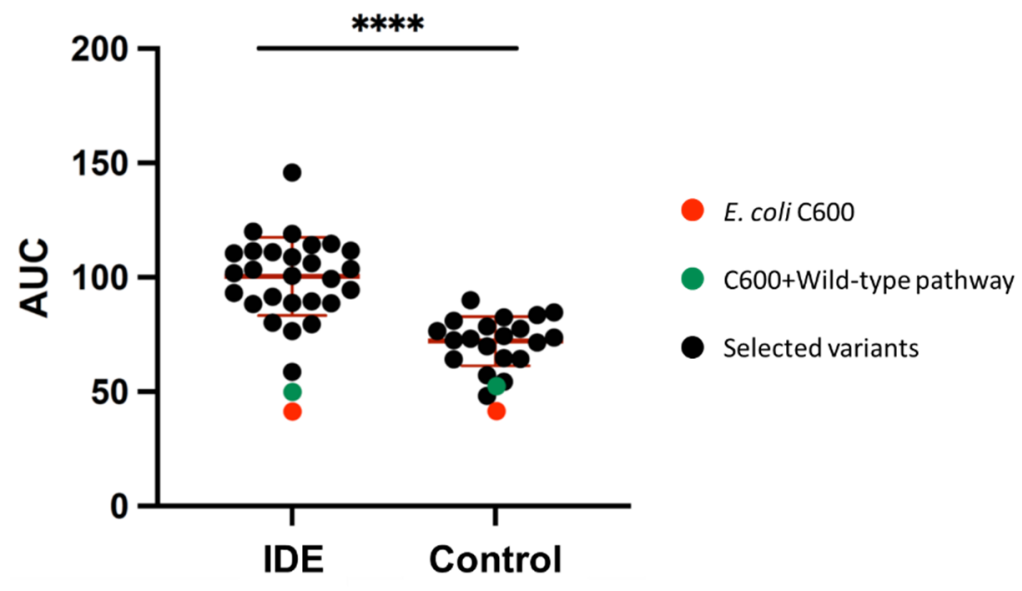

Figure S11. Comparing growth characteristics of variants isolated from IDE and Control tagatose selections. Variants from IDE and control selections were grown in tagatose minimal media and optical density was measured over time in a microplate reader. AUC (Area Under the Curve) is calculated by summing OD600 values obtained over the course of the experiment. ${ }^{* * * *}-p<10^{\wedge}-8$, Student's T test.

a

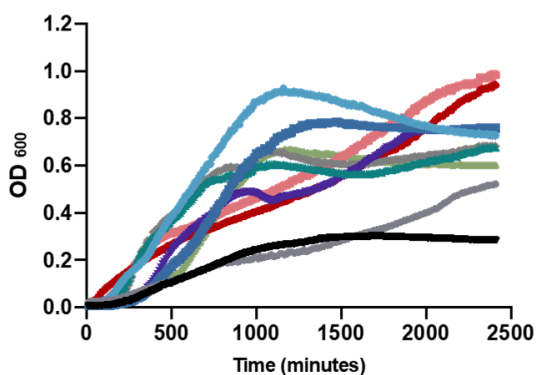

b

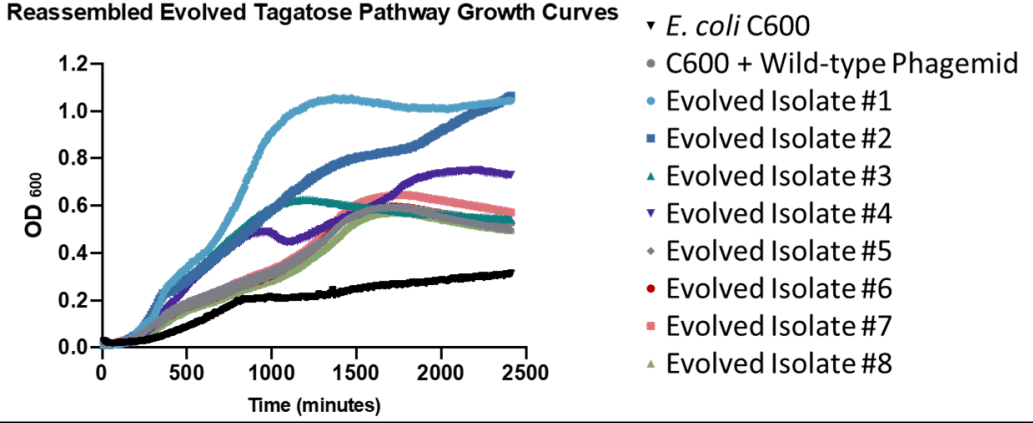

Figure S12. Growth curves of the selected tagatose variants. a. 8 variants were picked out of 27 isolates that were picked initially from tagatose agar plates and confirmed in plate reader. $\mathbf{b}$. Tagatose pathways from the 8 selected variants were assembled into unmutated backbone and grown in tagatose minimal media. 


\begin{tabular}{|c|c|c|c|}
\hline Amino Acid Change & Sequence & Gene & Isolate \\
\hline V56A & GTT --> GCT & orf48 & E3 \\
\hline Q127K & CAA --> AAA & orf48 & E1 \\
\hline RBS & A --> G & fruK2 & E3,E4 \\
\hline P47P & CCA --> CCC & fruK2 & E2 \\
\hline R149R & AGA --> AGG & fruK2 & E3 \\
\hline A183T & GCA --> ACA & fruA2 & E1 \\
\hline V428A & GTA --> GCA & fruA2 & E2 \\
\hline A39A & GCC --> GCT & gatY & E2 \\
\hline Y55D & TAT --> GAT & gatY & E2 \\
\hline P255L & CCA --> CTA & gatY & E1 \\
\hline
\end{tabular}

Table 2. Mutations detected in evolved tagatose consumption pathways. RBS indicates mutations were detected in the ribosome binding site. Grey filling indicates silent mutations. E\# indicates the number of the evolved isolate.

As microbial engineering moves toward applications demanding ever-higher performance (e.g. green production of fuels ${ }^{39}$ and chemicals ${ }^{40}$, sensing ${ }^{41}$ and biosynthesis on host-associated sites $^{42}$ ), the ability to engineer complex phenotypes is becoming increasingly important. Currently, optimizing the performance of multi-gene pathways is a challenging task. IDE offers the ability to perform directed evolution on long (at least up to $42 \mathrm{kbp}$ ) sequences of DNA with tunable error rates (up to $5.4^{*} 10^{-6}$ substitutions per bp per generation) and library sizes that scale trivially with culture volume (up to $4 \%$ of recipient cells contain mutants). We expect that the use of different mutagenesis methods (e.g. ultraviolet light and chemical mutagens) in place of a mutagenesis 234 plasmid can add further mutational flexibility. Importantly, the use of temperate phages (such as P1) to passage variants to fresh hosts greatly reduces the impact of off-target mutations and decouples mutagenesis and screening steps, providing a large degree of flexibility when designing selections. In particular, we expect this approach to be highly amenable to automation, enabling rapid and highly parallel evolution campaigns similar to the eVOLVER ${ }^{10}, \mathrm{PACE}^{18}$, and vehicles for directed evolution of complex phenotypes. 


\section{Methods:}

242

243

244

245

246

247

248

250

251

252

253

254

255

256

257

258

259

260

261

262

263

264

Strains and Media: E. coli Top10, NEB $5 \alpha$ and NEB $10 \beta$ were used for plasmid construction. E. coli C600 (CGSC 5394 C600) was used for optimization infection and packaging rates. E. coli Nissle 1917 and E. coli MG1655 were used to test IDE's applicability to different E. coli strains. All E. coli strains were grown in lysogeny broth (LB) (5g/L yeast extract, $10 \mathrm{~g} / \mathrm{L}$ tryptone, $10 \mathrm{~g} / \mathrm{L}$ $\mathrm{NaCl})$ at $37^{\circ} \mathrm{C}$ supplemented with ampicillin (Amp) $(100 \mu \mathrm{g} / \mathrm{mL})$, kanamycin $(\mathrm{Kan})(50 \mu \mathrm{g} / \mathrm{mL})$ or chloramphenicol $(\mathrm{Cm})(34 \mu \mathrm{g} / \mathrm{mL})$. Strains containing mutagenesis plasmids were grown in LB containing $1 \%(\mathrm{w} / \mathrm{v}) \mathrm{d}$-glucose and appropriate antibiotics. Bacillus licheniformis (ATCC® $14580^{\mathrm{TM}}$ ) was grown in Difco ${ }^{\mathrm{TM}}$ Nutrient Broth. All infection and packaging experiments were performed in Phage Lysate Media (PLM, LB with $100 \mathrm{mM} \mathrm{MgCl}_{2}$ and $5 \mathrm{mM} \mathrm{CaCl}_{2}$ ) or enhanced PLM (ePLM, LB $140 \mathrm{mM} \mathrm{MgCl} 2$ and $7 \mathrm{mM} \mathrm{CaCl}_{2}$ ). P1kc $\Delta$ coi::KanR and P1kc:10kb::KanR were gifts from Dr. Chase Beisel.

Preparation and transformation of electrocompetent cells: Overnight cultures of the desired strains were inoculated (1:100 dilution) to $50 \mathrm{~mL}$ LB media containing appropriate antibiotics and grown to OD 0.8 at $37^{\circ} \mathrm{C}$ and $250 \mathrm{rpm}$. Cells were then chilled on ice for 15 minutes before being pelleted by centrifugation at $3000 \mathrm{xg}$ for 5 minutes. Cells were then washed twice via resuspension in $25 \mathrm{~mL} \mathrm{10 \%} \mathrm{glycerol} \mathrm{and} \mathrm{pelleting} \mathrm{via} \mathrm{centrifugation} \mathrm{at} 3000 \mathrm{xg}$ for 5 minutes. Washed cells were then resuspended in $1 \mathrm{~mL} 10 \%$ glycerol and pelleted at $4000 \mathrm{xg}$ for 3 minutes in eppendorf microcentrifuge tubes. The resulting pellets were then resuspended in $0.5 \mathrm{~mL} 10 \%$ glycerol and divided into $50 \mu \mathrm{L}$ aliquots that were either used for transformation immediately or stored at $-80{ }^{\circ} \mathrm{C}$. Frozen cells were thawed on ice for 10 minutes before being used for transformation. Fresh cells yielded higher transformation efficiency. 
Cloning: Primers used in this study were obtained from Eurofins and are listed in Table S3. Plasmids and phagemids are listed in Table S4. NEBuilder ${ }^{\circledR}$ HiFi DNA Assembly Master Mix was used for plasmid and phagemid construction. SGI-DNA Gibson Assembly ${ }^{\circledR}$ (GA) HiFi 1-Step Kit assembly was used for construction of large phagemids. NEB Q $5^{\circledR}$ Site-Directed Mutagenesis Kit was used to introduce point mutations. Addgene \#40782 was used as the backbone for all phagemid cloning, and primers 1 and 2 are used to amplify this backbone for gibson cloning.

272 Construction of large phagemids: A $24.0 \mathrm{kbp}$ P1 phagemid (Addgene \#40784) was used as a backbone for constructing a $42 \mathrm{kbp}$ phagemid (PM-42.0). To construct this phagemid, 3 PCR reactions were used to amplify 5-7 kbp fragments from the Saccharomyces cerevisiae genome in addition to the phagemid backbone. Q5 ${ }^{\circledR}$ High-Fidelity $2 X$ Master Mix was used to amplify the parts and SGI-DNA Gibson Assembly ${ }^{\circledR}(\mathrm{GA}) \mathrm{HiFi}$ 1-Step Kit was used to assemble the parts. The assembled product was transformed into E. coli DH10B (ISA585). ZymoPURE ${ }^{\text {TM }}$ II Plasmid Midiprep Kit (Catalog No. D4200 \& D4201) was used to extract PM-42.0 from ISA585.

Phage Production: Overnight cultures of strains containing P1 and phagemid were subinoculated in ePLM (1:100 dilution) with appropriate antibiotics. At OD 0.8-1.0 cell cultures were induced with $20 \%$ L-arabinose (1/100 culture volume) and put back to the shaking incubator $\left(37^{\circ} \mathrm{C}, 250 \mathrm{rpm}\right)$. After 2 hours, the cultures were removed from the incubator and transferred to $15 \mathrm{~mL}$ centrifuge tubes containing chloroform. The tubes were left on ice for 5 minutes with gentle mixing or pipetting every minute. The tubes were then centrifuged at $3000 \mathrm{xg}$ for 10 minutes at $4{ }^{\circ} \mathrm{C}$. The produced phage (present in the supernatant) were then transferred to sterile tubes for 287 storage. Phage lysate is stable at $4{ }^{\circ} \mathrm{C}$ for 1 year and indefinitely at $-80{ }^{\circ} \mathrm{C}$.

289 Phage Infection: An overnight culture grown in LB with the appropriate antibiotics was 290 subinoculated into ePLM (1:100 dilution). At OD 1, the cells were spun down at $3000 \times 9$ for 5 
minutes. The supernatant was discarded and the pellet was resuspended in $1 / 3$ volume fresh ePLM.

292 The cells were then added to phage lysate in a $14 \mathrm{~mL}$ falcon culture tube. The infection mixture was incubated in a $37^{\circ} \mathrm{C}$ incubator (250 rpm shaking) for 20 minutes and then moved to a $37^{\circ} \mathrm{C}$ standing incubator for 20 minutes. The infection mixture was quenched with $1 \mathrm{~mL}$ of Super Optimal Broth (SOC) containing $200 \mathrm{mM}$ sodium citrate. The mixture was then incubated for 40 minutes in a $37{ }^{\circ} \mathrm{C}$ shaking incubator before being transferred to $50 \mathrm{~mL}$ LB media with the appropriate antibiotics or plated on LB agar plates containing the appropriate antibiotics.

Cell growth phase experiments: Three E. coli C600 colonies grown overnight in LB containing the appropriate antibiotics were subinoculated (1:100, 1:200 and 1:300 dilution) into three $50 \mathrm{~mL}$ PLM and grown to OD $0.5,1.0$ and 1.5 in shaking incubator $\left(37^{\circ} \mathrm{C} 250 \mathrm{rpm}\right)$. Cell cultures were harvested (3000 xg for 5 minutes) and resuspended with PLM to obtain the same cell count per C600 containing P1kc and $12 \mathrm{kbp}$ phagemid). Infected cultures were plated on $\mathrm{LB}+\mathrm{Cm}$ to count $\mathrm{CFU} / \mathrm{mL}$. then used to infect wild type E. coli $\mathrm{C} 600$ or E. coli C600 containing P1kc::10kb. Infected strains were plated on $\mathrm{LB}+\mathrm{Cm}$ plates to count $\mathrm{CFU} / \mathrm{mL}$.

Infecting different E. coli Strains: The $12 \mathrm{kbp}$ phagemid was transformed into E. coli C600,

314 MG1655, and Nissle containing P1kc:10kb::KanR. 3 biological replicates from the transformed 315 strains were grown overnight in $\mathrm{LB}+\mathrm{Cm}$ and used for phage production. Phage lysate from each 316 strain was used to infect wild type E. coli C600, MG1655, and Nissle. 
Flip recombinase to delete KanR from P1kc $\Delta$ coi::KanR and P1kc:10kb::KanR: Adapted from barricklab.org ${ }^{43}$. In brief, E. coli C600 containing $\mathrm{P} 1 \mathrm{kc} \Delta$ coi::KanR (ISA137) and P1kc:10kb::KanR (ISA138) were first transformed with pCP20 and plated in LB+Ampicillin agar plates and grown overnight at $30^{\circ} \mathrm{C}$. Grown colonies were inoculated into $1.0 \mathrm{~mL}$ of $\mathrm{LB}$ in eppendorf microcentrifuge tubes and grown overnight at $43^{\circ} \mathrm{C}$ to induce FLP recombinase expression and plasmid loss. Colonies were then screened for loss of KanR from the phage genome via plating in LB+Kan, LB+Amp and LB only plates.

Single and double Stop codon reversion: $A m p R$ was inserted into a phagemid backbone (ISA012) via Gibson assembly. Mutations were introduced via Q5 SDM to introduce either one or two stop codons in $C m R$. This phagemid, with a difunctional $C m R$, was transformed into $E$. coli C600 containing aTc-MP and P1 phage (ISA308, ISA311 and ISA363). 3 biological replicates were grown overnight in LB containing 1\% glucose, kanamycin, and ampicillin. The overnight

337 Random colonies were picked to verify stop codon reversion. 
342

343

344

345

346

347

(ISA384). In short, the p15A-sfGFP phagemid was mutated, transferred to fresh cells, and selected for higher fitness via outgrowth a total of 4 times. The phage lysates produced from each round's stationary phase cultures were used to infect wild type E. coli C600. These strains were analyzed via flow cytometry to compare their fluorescence to that of empty vector, wild-type, and cell cultures that passed through the steps with the exception of mutagenesis. Flow cytometry data was analyzed with FlowJo (FlowJo LLC). The sfGFP gene from 5-10 colonies was amplified via PCR and sequenced by Sanger sequencing to detect mutations.

\section{Improving pSC101-sfGFP fluorescence via IDE (see Supplementary Methods): The pSC101} origin and sfGFP were inserted into ISA012 via Gibson assembly. The resulting phagemid (pSC101-sfGFP) was then transformed into E. coli C600 containing aTc-MP and P1kc:10kb (ISA426). The resulting strain was mutated 2 times before selection for increased colony fluorescence. Colonies that exhibited higher GFP fluorescence were visually identified and analyzed via flow cytometry to quantify GFP production. Mutations that were found in pSC101 were introduced to the unevolved phagemid via Q5 SDM, transformed into E. coli C600, and analyzed via flow cytometry to confirm their causal effects.

Tagatose evolution (see Supplementary Methods): The tagatose pathway from Bacillus

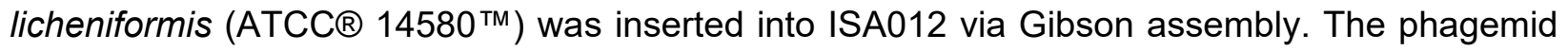
was then transformed into E. coli $\mathrm{C} 600$ containing aTc-MP and $\mathrm{P} 1 \mathrm{kc}: 10 \mathrm{~kb}$. The resulting strain was evolved through two rounds of diversification and selection as shown in Figure S12. The resulting phage lysate was used to infect wild-type E. coli $\mathrm{C600}$, and the resulting cells were plated on tagatose minimal media agar plates. Large colonies were picked and grown overnight in LB media containing chloramphenicol. The cultures were then washed with tagatose minimal media and grown for 40 hours in a plate reader (see methods: growth curves). The pathways in the colonies with faster growth rates were sequenced via Sanger sequencing. Resulting mutations 
were then reintroduced to unevolved phagemid via Q5 SDM and analyzed again via growth in tagatose minimal media.

Tagatose Minimal Media: The selection media for the tagatose minimal media was adapted from Van der Heiden, et al. (2013) $)^{37}$ with few modifications for E. coli C600. 20x Salt solution was composed of $\mathrm{KH}_{2} \mathrm{PO} 4(54.4 \mathrm{~g}), \mathrm{K}_{2} \mathrm{HPO}_{4}(208.8 \mathrm{~g})$, and $\mathrm{NH}_{4} \mathrm{Cl}(12 \mathrm{~g})$ for $1 \mathrm{~L}$ solution. $500 x$ mineral solution was composed of $\mathrm{MgCl}_{2} \cdot 6 \mathrm{H}_{2} \mathrm{O}(1 \mathrm{~g}), \mathrm{CaCl}_{2} \cdot 2 \mathrm{H}_{2} \mathrm{O}(0.25 \mathrm{~g}), \mathrm{FeCl}_{2} \cdot 4 \mathrm{H}_{2} \mathrm{O}(25 \mathrm{mg})$, $\mathrm{ZnSO}_{4} \cdot 7 \mathrm{H}_{2} \mathrm{O}(25 \mathrm{mg}), \mathrm{CoCl}_{2} \cdot \mathrm{H}_{2} \mathrm{O}(12.5 \mathrm{mg})$, CuSO $4 \cdot 5 \mathrm{H}_{2} \mathrm{O}(0.5 \mathrm{mg})$, and $\mathrm{MnSO}_{4} \cdot \mathrm{H}_{2} \mathrm{O}(0.14 \mathrm{~g})$ in $100 \mathrm{ml}$ solution. $100 x$ vitamin $\mathrm{mix}(50 \mathrm{ml})$ was composed of $5 \mathrm{mg}$ of thiamine- $\mathrm{HCl}$, nicotinic acid, folic acid, d-I-pantothenic acid, d-biotin, leucine, lysine, homoserine and riboflavin and $10 \mathrm{mg}$ of pyridoxal-HCl. $0.1 \%(\mathrm{wt} / \mathrm{vol})$ I-Casamino Acids and $1 \%(\mathrm{wt} / \mathrm{vol})$ tagatose were added to the minimal media.

\section{Growth curves:}

3 biological replicates of each isolate were grown overnight in 96-deep-well plates (VWR International, cat \#10755-248) in LB media. Saturated cultures in 96-deep-well were spun down at $2500 \mathrm{xg}$ for 10 minutes, the supernatant was discarded, and the pellet was resuspended in tagatose minimal media. These suspensions were spun again at $2500 \mathrm{xg}$ for 10 minutes, the supernatant was discarded, and the pellet was resuspended in tagatose minimal media one last time. This culture was then transferred to 96-well-plates (Costar, Corning ${ }^{\mathrm{TM}}$ 3788) containing tagatose minimal media (1:200 dilution) and grown for 40 hours in a plate reader (BioTek Synergy $^{\mathrm{TM}}$ H1, Shake Mode: Double Orbital, Orbital Frequency: continuous shake $365 \mathrm{cpm}$, Interval: 10 minutes).

\section{Acknowledgements:}


394 We thank members of the Crook Lab, and Daphne Collias, Dr. Chase Beisel, Dr. Jennie Fagen,

395 and Dr. Janetta Hakovirta for valuable discussions and input. We also thank the labs of Dr.

396 Christopher Anderson (UC Berkeley) for phagemid constructs (Addgene \#40782, \#40783 and

397 \#40784), Dr. David R. Liu (Harvard University) for the MP6 plasmid (Addgene \#69669), and Dr.

398 Chase Beisel for wild type and engineered P1 bacteriophages. This work was supported by

399 startup funds from North Carolina State University's Chemical and Biomolecular Engineering

400 (CBE) Department and NCSU's Faculty Research and Professional Development Fund. I.S.A. is

401 supported by NCSU CBE startup funds and the Ministry of Higher Education - Oman.

402

403 Author contributions

404 I.S.A. and N.C. designed and conceived the study. I.S.A. and D.J.H. conducted all experiments.

405 N.C. supervised the research. I.S.A., D.J.H., and N.C. wrote the manuscript.

406

407 Competing interests

408 I.S.A. and N.C have filed a patent application related to this work.

409

410 References:

411 1. Harvey, A. L., Edrada-Ebel, R. \& Quinn, R. J. The re-emergence of natural products for drug 412 discovery in the genomics era. Nature Reviews Drug Discovery vol. 14 111-129 (2015).

413 2. Crook, N. \& Alper, H. S. Classical Strain Improvement. Engineering Complex Phenotypes in $414 \quad$ Industrial Strains 1-33 (2012) doi:10.1002/9781118433034.ch1.

415 3. Lee, S. Y. et al. A comprehensive metabolic map for production of bio-based chemicals. $416 \quad$ Nature Catalysis 2, 18-33 (2019). 
4. Pham, H. L. et al. Engineering a riboswitch-based genetic platform for the self-directed evolution of acid-tolerant phenotypes. Nat. Commun. 8, 411 (2017).

5. Crook, N. et al. Adaptive Strategies of the Candidate Probiotic E. coli Nissle in the Mammalian Gut. Cell Host Microbe 25, 499-512.e8 (2019).

6. Farkas, Z. et al. Hsp70-associated chaperones have a critical role in buffering protein production costs. Elife 7, (2018).

7. Tsoi, R. et al. Metabolic division of labor in microbial systems. Proc. Natl. Acad. Sci. U. S. A. 115, 2526-2531 (2018).

8. Rosano, G. L. \& Ceccarelli, E. A. Recombinant protein expression in Escherichia coli: advances and challenges. Front. Microbiol. 5, 172 (2014).

9. Gleizer, S. et al. Conversion of Escherichia coli to Generate All Biomass Carbon from CO2. Cell 179, 1255-1263.e12 (2019).

10. Wong, B. G., Mancuso, C. P., Kiriakov, S., Bashor, C. J. \& Khalil, A. S. Precise, automated control of conditions for high-throughput growth of yeast and bacteria with eVOLVER. Nat. Biotechnol. 36, 614-623 (2018).

11. Georg, J. \& Hess, W. R. cis-antisense RNA, another level of gene regulation in bacteria. Microbiol. Mol. Biol. Rev. 75, 286-300 (2011).

12. Meng, J. et al. A genome-wide inducible phenotypic screen identifies antisense RNA constructs silencing Escherichia coli essential genes. FEMS Microbiol. Lett. 329, 45-53 (2012).

13. Bikard, D., Hatoum-Aslan, A., Mucida, D. \& Marraffini, L. A. CRISPR interference can prevent natural transformation and virulence acquisition during in vivo bacterial infection. Cell Host Microbe 12, 177-186 (2012).

14. Reis, A. C. et al. Simultaneous repression of multiple bacterial genes using nonrepetitive extra-long sgRNA arrays. Nat. Biotechnol. 37, 1294-1301 (2019). 
15. Dong, C., Fontana, J., Patel, A., Carothers, J. M. \& Zalatan, J. G. Synthetic CRISPR-Cas gene activators for transcriptional reprogramming in bacteria. Nat. Commun. 9, 2489 (2018).

16. Roth, T. B., Woolston, B. M., Stephanopoulos, G. \& Liu, D. R. Phage-Assisted Evolution of Bacillus methanolicus Methanol Dehydrogenase 2. ACS Synth. Biol. 8, 796-806 (2019).

17. Suzuki, T. et al. Crystal structures reveal an elusive functional domain of pyrrolysyl-tRNA synthetase. Nat. Chem. Biol. 13, 1261-1266 (2017).

18. Esvelt, K. M., Carlson, J. C. \& Liu, D. R. A system for the continuous directed evolution of biomolecules. Nature 472, 499-503 (2011).

19. Brödel, A. K., Rodrigues, R., Jaramillo, A. \& Isalan, M. Accelerated evolution of a minimal 63-amino acid dual transcription factor. Sci Adv 6, eaba2728 (2020).

20. DeBenedictis, E. A., Chory, E. J., Gretton, D., Wang, B. \& Esvelt, K. A high-throughput platform for feedback-controlled directed evolution. bioRxiv 2020.04.01.021022 (2020) doi:10.1101/2020.04.01.021022.

21. Competent Cell Essentials-10 Molecular Cloning Strategies - US.

22. Johnston, C. W., Badran, A. H. \& Collins, J. J. Continuous bioactivity-dependent evolution of an antibiotic biosynthetic pathway. Nat. Commun. 11, 4202 (2020).

23. Coren, J. S., Pierce, J. C. \& Sternberg, N. Headful packaging revisited: the packaging of more than one DNA molecule into a bacteriophage P1 head. J. Mol. Biol. 249, 176-184 (1995).

24. Kittleson, J. T., DeLoache, W., Cheng, H.-Y. \& Anderson, J. C. Scalable plasmid transfer using engineered P1-based phagemids. ACS Synth. Biol. 1, 583-589 (2012).

25. Badran, A. H. \& Liu, D. R. Development of potent in vivo mutagenesis plasmids with broad mutational spectra. Nat. Commun. 6, 8425 (2015).

26. Westwater, C., Schofield, D. A., Schmidt, M. G., Norris, J. S. \& Dolan, J. W. Development of a P1 phagemid system for the delivery of DNA into Gram-negative bacteria. Microbiology 148, 943-950 (2002). 
27. Coughlin, R. T., Tonsager, S. \& McGroarty, E. J. Quantitation of metal cations bound to membranes and extracted lipopolysaccharide of Escherichia coli. Biochemistry 22, 20022007 (1983).

28. Cvirkaite-Krupovic, V., Krupovic, M., Daugelavicius, R. \& Bamford, D. H. Calcium iondependent entry of the membrane-containing bacteriophage PM2 into its Pseudoalteromonas host. Virology 405, 120-128 (2010).

29. Tomás, J. M. \& Kay, W. W. Effect of bacteriophage P1 lysogeny on lipopolysaccharide composition and the lambda receptor of Escherichia coli. J. Bacteriol. 159, 1047-1052 (1984).

30. Kasman, L. M. et al. Overcoming the phage replication threshold: a mathematical model with implications for phage therapy. J. Virol. 76, 5557-5564 (2002).

31. Taylor-Parker, J. Plasmids 101: E. coli Strains for Protein Expression. https://blog.addgene.org/plasmids-101-e-coli-strains-for-protein-expression.

32. Kafri, M., Metzl-Raz, E., Jona, G. \& Barkai, N. The Cost of Protein Production. Cell Rep. 14, 22-31 (2016).

33. Fu, J. L., Kanno, T., Liang, S.-C., Matzke, A. J. M. \& Matzke, M. GFP Loss-of-Function Mutations in Arabidopsis thaliana. G3 5, 1849-1855 (2015).

34. Furuno, S., Watanabe-Murakami, Y., Takebe-Suzuki, N. \& Yamaguchi, K. Negative control of plasmid pSC101 replication by increased concentrations of both initiator protein and iterons. J. Gen. Appl. Microbiol. 46, 29-37 (2000).

35. Thompson, M. G. et al. Isolation and characterization of novel mutations in the pSC101 origin that increase copy number. Sci. Rep. 8, 1590 (2018).

36. Pédelacq, J.-D., Cabantous, S., Tran, T., Terwilliger, T. C. \& Waldo, G. S. Engineering and characterization of a superfolder green fluorescent protein. Nat. Biotechnol. 24, 79-88 (2006).

37. Van der Heiden, E. et al. A pathway closely related to the (D)-tagatose pathway of gramnegative enterobacteria identified in the gram-positive bacterium Bacillus licheniformis. Appl. Environ. Microbiol. 79, 3511-3515 (2013). 
494 38. Crook, N. et al. In vivo continuous evolution of genes and pathways in yeast. Nat. Commun.

$495 \quad 7,13051(2016)$.

496 39. Gronenberg, L. S., Marcheschi, R. J. \& Liao, J. C. Next generation biofuel engineering in 497 prokaryotes. Curr. Opin. Chem. Biol. 17, 462-471 (2013).

498 40. Beller, H. R., Lee, T. S. \& Katz, L. Natural products as biofuels and bio-based chemicals:

499 fatty acids and isoprenoids. Nat. Prod. Rep. 32, 1508-1526 (2015).

500 41. Daeffler, K. N.-M. et al. Engineering bacterial thiosulfate and tetrathionate sensors for 501 detecting gut inflammation. Mol. Syst. Biol. 13, 923 (2017).

502 42. Fischbach, M. A. \& Segre, J. A. Signaling in Host-Associated Microbial Communities. Cell $503 \quad 164,1288-1300(2016)$.

504

43. Barrick

$\mathrm{Lab}$

$::$

ProcedureFLPFRTRecombination. 